\title{
The simultaneous effect of electromagnetic and ultrasound treatments on Escherichia coli count in red grape juice
}

\author{
Bahram Hosseinzadeh Samani ${ }^{1}$, Zahra Lorigooini ${ }^{2}$, Sajad Rostami ${ }^{1}$, Hemad Zareiforoush ${ }^{3}$, Mehrsa Behruzian $^{1}$, \\ Ava Behruzian ${ }^{1}$ \\ ${ }^{1}$ Department of Mechanical Engineering of Biosystem, Shahrekord University, Iran \\ ${ }^{2}$ Medical Plants Research Center, Basic Health Sciences Institute, Shahrekord University of Medical Sciences, Shahrekord, Iran \\ ${ }^{3}$ Department of Agricultural Mechanization Engineering, University of Guilan, Rasht, Iran
}

\section{A R T I C L E I N F O}

Article Type:

Original Article

\section{Article History:}

Received: 23 May 2017

Accepted: 19 November 2017

\section{Keywords:}

Pasteurization

Cavitation

Ultrasonic

Temperature

Grape juice

\begin{abstract}
A B S T R A C T
Introduction: The thermal pasteurization is a common method for maintaining fruit juice and increasing shelf life, but the thermal processing changes the flavor and color of the products. The aim of this study was to investigate the effect of a new method of combining heat and ultrasound on the number of the Escherichia coli present in grape juice.

Methods: In this study, the effects of the microwave power, temperature, ultrasound power and ultrasonic exposure time were evaluated on $E$. coli count of red grape juice. In order to determine the microbial inactivation by microwave and ultrasound, E. coli at a concentration of $6 \times 10^{6}$ per mL was inoculated to red grape juice.

Results: The effects of microwave power, grape juice temperature, ultrasound power and ultrasonic exposure time on the reduction of $E$. coli were significant $(P<0.05)$. The model showed that in reducing $E$. coli the importance of the final temperature of the juice was higher than the microwave power. In addition, the ultrasonic power was more effective in E. coli reduction as compared to the microwave power.

Conclusion: Both sample temperature and ultrasonic duration were important independent variables and effective factors on $E$. coli reduction.
\end{abstract}

Implication for health policy/practice/research/medical education:

Considering the benefits of grape juice, including blood purification, treatment of lung, kidney and skin diseases, its pasteurization processing and production are of special importance. Pasteurize grape juice in optimal conditions, a short time and better quality using a system consisting of microwave and ultrasound device to reduce the harmful effects of high temperatures will be valuable for the food industry.

Please cite this paper as: Hosseinzadeh Samani B, Lorigooini Z, Rostami S, Zareiforoush H, Behruzian M, Behruzian A. The simultaneous effect of electromagnetic and ultrasound treatments on Escherichia coli count in red grape juice. J Herbmed Pharmacol. 2018;7(1):29-36. doi: 10.15171/jhp.2018.06

\section{Introduction}

Nowadays in the food industry in order to deactivate enzymes and microorganisms and increase a shelf life of fruit juices, the high temperature and the short time in pasteurization is used. The high temperature of pasteurization has the undesirable effects on nutrients and the nutritional value of food, including the loss of vitamins, non-enzymatic browning, transformed protein and the loss of taste food $(1,2)$. As mentioned, all thermal methods give rise to reducing the qualitative properties (vitamins, anthocyanin, etc) of fruit juices. For this purpose, researchers have been looking for solutions that by combining new thermal and non-thermal approaches, the damaging effects of conventional pasteurization be removed. The proposed approach can be non-thermal methods such as ultrasound waves, high hydrostatic pressure, electric field and the mixed methods (3-7). Usually, in some new ways, the nature of the effect of heat is changed. For example, the microwave and Ohmic methods are used for heating the products. These methods may have different effects on the pasteurizing products as compared with other methods because of their heating 
procedure $(8,9)$. The ultrasound is one of the non-thermal methods suggested in the literature. Using high-intensity ultrasound in the industrial processing is generally based on the proper use of a series of mechanisms activated by the ultrasonic energy, such as heat, agitation, diffusion, interface instabilities, friction and chemical effects (10). Researchers analyzed the effects of ultrasound waves and mild heat on the active and passive microorganisms of orange juice during treatment and after treatment. They found that the pulp of fruit juice increased the microbes' resistance to the ultrasound waves. In addition, they proposed the use of ultrasound combined with other approaches to reduce the microbial activity and increase storage time (11).

In a study, the treatments of tomato juice using the thermal ultrasound and conventional heating at the same temperature of ultrasound were compared. The results showed that for inactivating pectin methylesterase by $90 \%$ the amounts of time in applied ultrasound were 41.8, 11.7 and 4.3 minutes and in the case of thermal, conventional treatments were 90.1, 23.5 and 3.5 minutes (12).

One of the thermal methods, having a different mechanism compared to the direct thermal method, is the use of microwaves. Basically the microwave waves with wavelengths between 0.001 and $1 \mathrm{~m}$, and a frequency of approximately $300-300000 \mathrm{MHz}$, are part of the electromagnetic spectrum. Since the direction of the magnetic field at the frequencies used millions of times per second is changed, therefore the molecules that have a polar nature, are influenced by such fluctuations and for coordination with the fluctuations swing their poles. This permanent swing gives rise to producing friction and heat, which the heating mechanism is different from the conventional thermal methods $(3,13)$.

According to the literature, studies on the use of such methods in order to eliminate microorganisms have been performed. However, evaluating the effect of ultrasound and microwave waves has recently been considered simultaneously $(2,10)$. In this study, we tried using a system consisting of microwave and ultrasound device to reduce the harmful effects of high temperatures and Pasteurize grape juice in optimal conditions, a short time and better quality.

One of welcome fruit juices is grape juice. It has many properties, including blood purification, treatment of lung, kidney and skin diseases. Considering the benefits and importance of fruit juices, especially grape juice, its processing and production are of special importance. Hence, the aim of this study was to evaluate the effect of the novel mixed method of heat-ultrasound on the number of Escherichia coli in the grape juice.

\section{Material and Method}

\section{Sample preparation}

In this study, an ultrasound generator with a maximum power of $400 \mathrm{~W}$ and a frequency of $20 \pm 0.5 \mathrm{kHz}$, made in Ultrasonic Technology Development Co. in Iran, was used. First, a certain amount of red grapes was prepared from the local markets. After rinsing, drying, and seeds removing, grape juice was extracted with an electric juicer. In order to separate the suspended pulp particles and tissue components, the extracted juice was centrifuged for 20 minutes at $6000 \mathrm{rpm}$. To fully separate the remaining suspended particles, the transparent part of the extract was passed through a paper filter using a vacuum pump (10). Then, the samples were poured into a reactor with diameter and height of 80 and $50 \mathrm{~mm}$, respectively. It should be noted that the dimensions of the reactor in pretrials were optimized. The schematic of the device used is presented in Figure 1.

\section{Preparation of microbial suspensions}

Once the lyophilized standard strain of E. coli vial was opened, it was cultivated on an agar nutrient culture. Therefore, a loop of grown microbial strain on agar was inoculated to a $25 \mathrm{~mL}$ nutrient liquid culture under sterile conditions in order to prepare the microbial suspension. This was kept in an oven for $18-24$ hours at $37^{\circ} \mathrm{C}$. The liquid culture containing the grown cells was centrifuged for 5 minutes at $8000 \mathrm{rpm}$ (7656 g) so that the resulting cellular mass was suspended in sterile grape juice. A total of $3 \mathrm{~mL}$ of this suspension was inoculated to $300 \mathrm{~mL}$ of sour cherry juice. For adaptation purpose, it was held for 15-30 minutes before the deactivation. It should be noted that the cultures used for primary activation and proliferation included nutrient solid/liquid cultures (14). It is worth noting that microbial level of E. coli (PTCC 5052) was obtained from Iran Industrial and Scientific Research Organization.

Processing methods and statistical analysis The residual number of $E$. coli and energy consumption for reducing one cycle of $E$. coli were defined as two

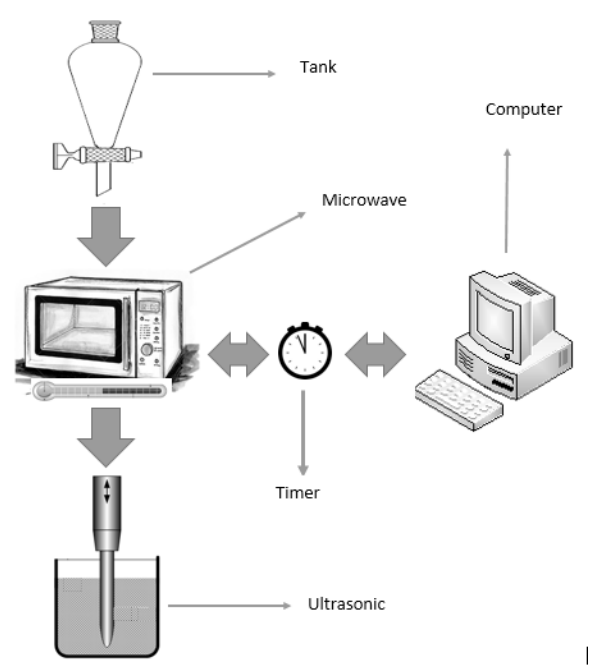

Figure 1. Schematic of the designed microwave-ultrasound processing system. 
Table 1. Experimental range and levels of independent variables

\begin{tabular}{|c|c|c|c|c|c|}
\hline \multirow{2}{*}{ Independent variable } & \multicolumn{5}{|c|}{ Range of level } \\
\hline & $-\alpha$ & -1 & 0 & 1 & $+\alpha$ \\
\hline Microwave power (W) & 200 & 350 & 500 & 650 & 800 \\
\hline Temperature $\left({ }^{\circ} \mathrm{C}\right)$ & 20 & 30 & 40 & 50 & 60 \\
\hline Ultrasound power (W) & 80 & 160 & 240 & 320 & 400 \\
\hline Ultrasonic exposure time (min) & 2 & 3 & 4 & 5 & 6 \\
\hline
\end{tabular}

dependent variables and the ultrasonic power, ultrasonic exposure time, microwave power and temperature of the sample were considered as independent variables. In this study, using the response surface method (RSM) and the central composite design (Table 1) the optimal conditions to minimize microorganisms and energy consumption of grape juice during the pasteurization process were determined.

To carry out the experiments, first, the inoculated grape juice was poured into the system tank. The experimental treatments were set according to Table 1. Then, by adjusting a level meter, $200 \mathrm{~mL}$ of grape juice was obtained for each test. The sample was heated in a microwave device to the desired temperature, and then, it was poured into the ultrasound reactor. During treating with ultrasound the temperature of the sample was fixed by the laboratory equipment. Finally, the treated sample to determine the amount of reduced E. coli was removed from the system. It should be noted that in all phases of the experiment, the power consumed by a watt meter (TROTEC, BX11 model) was recorded.

\section{Results}

As analysis of variance (ANOVA) for quadratic model shows, the model of data obtained from the reduction of E. coli in the sample of the fruit juice was significant. In addition, the significant lack of fit index also indicated the efficiency of the resulting model (Table 2). Adjusted R-squared and coefficient of variation (CV) for the model were equal to 0.97 and 2.25 , respectively.

The results of ANOVA for the amount of E. coli (Table 2) show that, except for the coefficient multiplied by the ultrasonic power and time, other coefficients of variables in the model are statistically significant at the $10 \%$ level. As seen, some coefficients of the model were not significant and were removed to simplify the fitted model. As a result, the final equation was obtained as equation (5).

$\log \left(\mathrm{N} / \mathrm{N}_{0}\right)=2.2007+3.54240 \mathrm{E}-003 * \mathrm{MP}-0.07098 \quad 4 * \mathrm{~T}$ $-0.027299 * \mathrm{UP}-0.23108 * \mathrm{t}-6.77205 \mathrm{E}-005 * \mathrm{MP} * \mathrm{~T}$ $+7.76669 \mathrm{E}-006 * \mathrm{MP} * \mathrm{UP}+5.96446 \mathrm{E}-005 * \mathrm{MP} * \mathrm{t}$ $+1.38662 \mathrm{E}-004 * \mathrm{~T} * \mathrm{UP}+4.52326 \mathrm{E}-003 * \mathrm{~T} * \mathrm{t}+7.14539 \mathrm{E}-$ $004 * \mathrm{UP}^{*} \mathrm{t}-2.91249 \mathrm{E}-006 * \mathrm{MP}^{2}+2.73002 \mathrm{E}-004 * \mathrm{~T}^{2}+$ $2.37533 \mathrm{E}-005 * \mathrm{UP}^{2}-0.026152 * \mathrm{t}^{2}$

And the final equation in terms of coded factors was obtained as follows:

$\log \left(\mathrm{N} / \mathrm{N}_{0}\right)=-3.25+3.553 \mathrm{E}-003 * \mathrm{MP}-0.32 * \mathrm{~T}-0.29$
Table 2. ANOVA of the coefficients of quadratic model in the response surface method for the amount of $E$. coli

\begin{tabular}{lccc}
\hline Source & $d f$ & Mean square & $\boldsymbol{P}$ value \\
\hline Model & 14 & 5.95 & 0.0001 \\
Microwave power & 1 & $3.027 \mathrm{E}-004$ & 0.8110 \\
Temperature & 1 & 2.4 & 0.0001 \\
Ultrasonic power & 1 & 2.24 & 0.0001 \\
Time & 1 & 0.081 & 0.0018 \\
Microwave power* Temperature & 1 & 0.17 & 0.0001 \\
Microwave power* Ultrasonic power & 1 & 0.14 & 0.0002 \\
Microwave power* Time & 1 & $1.281 \mathrm{E}-003$ & 0.6242 \\
Temperature* Ultrasonic power & 1 & 0.20 & 0.0001 \\
Temperature* Time & 1 & 0.033 & 0.0258 \\
Ultrasonic power* Time & 1 & 0.052 & 0.0075 \\
Microwave power* Microwave power & 1 & 0.075 & 0.0023 \\
Temperature* Temperature & 1 & 0.013 & 0.1341 \\
Ultrasonic power* Ultrasonic power & 1 & 0.46 & 0.0001 \\
Time* Time & 1 & 0.012 & 0.1498 \\
Residual & 12 & 0.061 & \\
Lack of Fit & 10 & 0.057 & 0.9918 \\
Pure Error & 2 & $3.596 \mathrm{E}-003$ & \\
Cor Total & 26 & 6.01 & \\
\hline
\end{tabular}

* UP $-0.058 * \mathrm{t}-0.10 * \mathrm{MP} * \mathrm{~T}+0.093 * \mathrm{MP} * \mathrm{UP}+$ $8.974 \mathrm{E}-003 * \mathrm{MP} * \mathrm{t}+0.11 * \mathrm{~T} * \mathrm{UP}+0.045 * \mathrm{~T} * \mathrm{t}+0.57$ $* \mathrm{UP} * \mathrm{t}-0.066 * \mathrm{MP}^{2}+0.027 * \mathrm{~T}^{2}+0.15 * \mathrm{UP}^{2}-0.026 *$ $\mathrm{t}^{2}$

Where $\mathrm{N}_{0}$ and $\mathrm{N}$ were primary and secondary counts, MP was microwave power (W), $\mathrm{T}$ was the sample temperature $\left({ }^{\circ} \mathrm{C}\right)$, UP was ultrasonic power $(\mathrm{W})$, and $\mathrm{t}$ was the ultrasonic exposure time (min). As equations show the temperature is more important than other parameters on E. coli reduction.

Figure 2 shows the proper adaptation of experimental data and data obtained from the model.

Also, the analysis of variance results for the second order model shows that data from the $E$. coli count reduction of 1 kJ energy consumption (was named ERE) was statistically significant. The adjusted coefficient of determination and CV of the model were 0.99 and 4.6 , respectively. The results of the ANOVA for cycle/energy are shown in Table 3. The final relations in terms of the actual factors and coded factors were obtained as equations 7 and 8, respectively:

$\mathrm{ERE}=+0.28935-6.76471 \mathrm{E}-004 * \mathrm{MP}-5.54457 \mathrm{E}-003 *$ $\mathrm{T}+1.21206 \mathrm{E}-003 * \mathrm{UP}+8.17251 \mathrm{E}-003 * \mathrm{t}+5.57457 \mathrm{E}-$ 


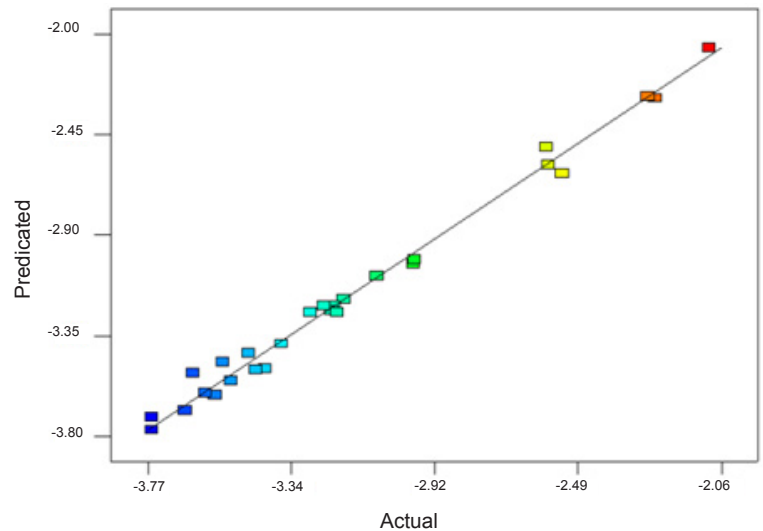

Figure 2. Fitting experimental data vs. the data of the model for the reduction of $E$. coli count.

Table 3. ANOVA results for second order coefficients using response surface method for energy consumption (ERE)

\begin{tabular}{lccc}
\hline Source & $d f$ & Mean square & $P$ value \\
\hline Model & 14 & $3.107 \mathrm{E}-003$ & 0.0001 \\
Microwave power & 1 & 0.027 & 0.0001 \\
Temperature & 1 & $8.045 \mathrm{E}-003$ & 0.0001 \\
Ultrasonic power & 1 & $3.096 \mathrm{E}-003$ & 0.0001 \\
Time & 1 & $1.101 \mathrm{E}-004$ & 0.0573 \\
Microwave power* Temperature & 1 & $1.119 \mathrm{E}-003$ & 0.0001 \\
Microwave power* Ultrasonic power & 1 & $7.276 \mathrm{E}-004$ & 0.0002 \\
Microwave power* Time & 1 & $9.054 \mathrm{E}-006$ & 0.5580 \\
Temperature* Ultrasonic power & 1 & $7.524 \mathrm{E}-004$ & 0.0001 \\
Temperature* Time & 1 & $5.558 \mathrm{E}-005$ & 0.1612 \\
Ultrasonic power* Time & 1 & $6.911 \mathrm{E}-005$ & 0.1218 \\
Microwave power* Microwave power & 1 & $1.328 \mathrm{E}-003$ & 0.0001 \\
Temperature* Temperature & 1 & $3.811 \mathrm{E}-004$ & 0.0021 \\
Ultrasonic power* Ultrasonic power & 1 & $4.318 \mathrm{E}-004$ & 0.0013 \\
Time* Time & 1 & $2.833 \mathrm{E}-005$ & 0.3074 \\
Residual & 12 & $2.493 \mathrm{E}-005$ & \\
Lack of Fit & 10 & $2.960 \mathrm{E}-005$ & 0.6234 \\
Pure Error & 2 & $1.576 \mathrm{E}-006$ & \\
Cor Total & 26 & 6.01 & \\
\hline
\end{tabular}

$006 * \mathrm{MP} * \mathrm{~T}-5.61955 \mathrm{E}-007 * \mathrm{MP} * \mathrm{UP}-5.01495 \mathrm{E}-006$ $* \mathrm{MP} * \mathrm{t}-8.57208 \mathrm{e}-006 * \mathrm{~T} * \mathrm{UP}-1.86384 \mathrm{E}-004 * \mathrm{~T} * \mathrm{t}-$ $2.59792 \mathrm{E}-005 * \mathrm{UP} * \mathrm{t}+3.86676 \mathrm{E}-007 * \mathrm{MP}^{2}+4.66153 \mathrm{E}-$ $005 * \mathrm{~T}^{2}-7.29168 \mathrm{E}-007 * \mathrm{UP}^{2}+1.27095 \mathrm{E}-003 * \mathrm{t}^{2}$

$\mathrm{ERE}=+0.099-0.033 * \mathrm{MP}-0.018 * \mathrm{~T}+0.011 * \mathrm{UP}+$ $2.142 \mathrm{E}-003 * \mathrm{t}+8.362 \mathrm{E}-003 * \mathrm{MP} * \mathrm{~T}-6.743 \mathrm{E}-003 *$ $\mathrm{MP} * \mathrm{UP}-7.522 \mathrm{E}-004 * \mathrm{MP} * \mathrm{t}-6.858 \mathrm{E}-003 * \mathrm{~T} * \mathrm{UP}-$ $1.864 \mathrm{E}-003 * \mathrm{~T} * \mathrm{t}-2.078 \mathrm{E}-003 * \mathrm{UP} * \mathrm{t}+8.700 \mathrm{E}-003 *$ $\mathrm{MP}^{2}+4.662 \mathrm{E}-003 * \mathrm{~T}^{2}-4.667 \mathrm{E}-003 * \mathrm{UP}^{2}+1.271 \mathrm{E}-003$ $* \mathrm{t}^{2}$

Compared to actual factors and coded factors, microwave power is more contributed to ERE.

Figure 3 shows fitting of experimental data and model data

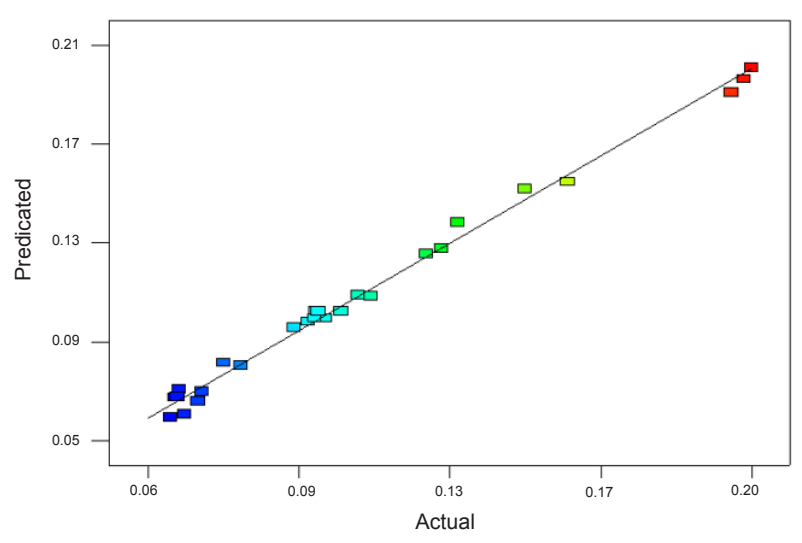

Figure 3. Relation between experimental data and model data to Energy consumption.

for E. coli count reduction of $1 \mathrm{~kJ}$ energy consumption. The importance of the final temperature of grape juice in reducing $E$. coli is higher than the microwave power that causes the temperature. The increase in the temperature led to an increase in the slope of the E. coli reduction. However, the increased microwave power had a lower slope than the temperature. At low temperatures, increasing the microwave power had no effect on the reduction of $E$. coli. Equation 5 shows that the product of the microwave power and temperature is a negative coefficient, indicating that by increasing either of two independent variables referenced, the result of the equation 5 would be a larger negative number, indicating a greater reduction in E. coli. Furthermore, the effect of the microwave power on ERE was more than the effect of temperature. As shown in Figure $4 \mathrm{~b}$, increase in the microwave power from $350 \mathrm{~W}$ to $650 \mathrm{~W}$ in a cycle led to $50 \%$ reduction in ERE, while increasing the temperature from $30^{\circ} \mathrm{C}$ to $50^{\circ} \mathrm{C}$ resulted in $40 \%$ reduction in ERE.

The ultrasound power had a higher impact on E. coli reduction in grape juice as compared to the microwave power (Figures 4c). Increasing the ultrasonic power from $160 \mathrm{~W}$ to $320 \mathrm{~W}$ caused $80 \%$ increase in ERE, but increasing the microwave power led to $50 \%$ decrease in ERE. Hence, it can be concluded that reducing the number of microorganisms in a cycle for each $\mathrm{kJ}$ of ultrasound energy consumption has been increased as compared to microwave method (Figure $4 \mathrm{~d}$ ).

As shown in Figures $4 \mathrm{c}, 4 \mathrm{e}$ and $4 \mathrm{~g}$, by increasing the ultrasonic power and the irradiation time, the slope of $E$. coli reduction is more than the situation in which the microwave power is increasing. By increasing the ultrasound exposing time the number of E. coli in grape juice decreases (Figure 4e).

Increased ultrasound energy consumption by reducing the microbial number in a cycle was more effective as compared to increasing the treatment time with ultrasound. It can be seen in Figure 4 that with doubling the ultrasound energy consumption, on average, a $30 \%$ 
increase in ERE was obtained, while with a two-fold increase in the treatment time no considerable change in ERE was observed. Both the sample temperature and the ultrasonic irradiation time are the important and effective independent variables in E. coli reduction (Figure $4 \mathrm{~g}$ ). An approximately two-fold increase in temperature has led to a $37 \%$ decrease in ERE. However, increased ultrasound power has the increasing slope. Hence, it can be said that increased ultrasound power can increase the system performance. The result can also be derived from equation 5 with respect to the negative sign of the coefficient of product for ultrasound power and temperature (Figure $4 \mathrm{~h}$ ).

Finally, the process was optimized. The objective function was obtained in the form of equation 5. The purpose of this optimization was to obtain the conditions of the independent variables (microwave power, sample temperature, ultrasound power and ultrasound treatment duration) in which the amount of E. coli in the sample would be equal to zero with the lowest energy consumption.

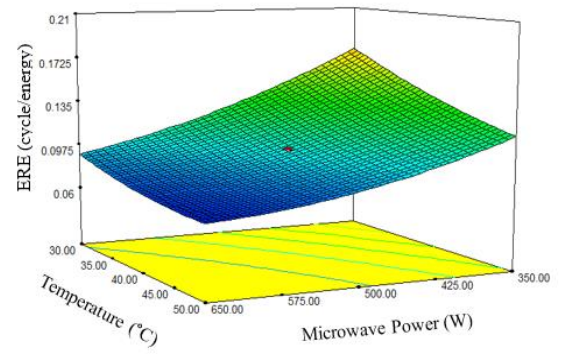

(a)

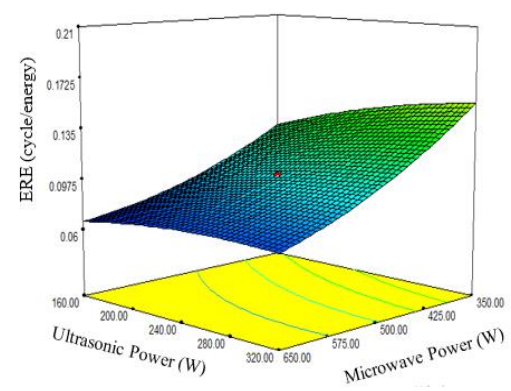

(c)

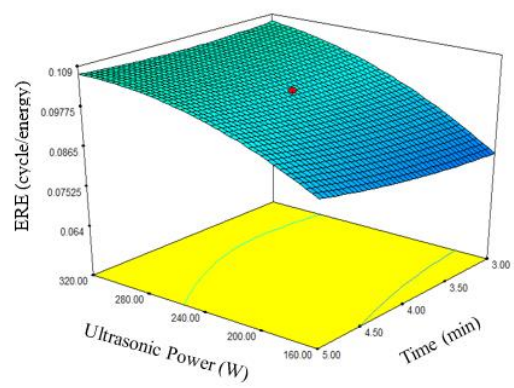

(e)
Another boundary condition for the optimization can be noted by the scope of the independent variables. In this study, the same experimental scope was considered. Table 4 shows the boundary conditions of process optimization: At the optimum point, microwave power, sample temperature, ultrasound power and ultra-sonication time were equal to $350 \mathrm{~W}, 30{ }^{\circ} \mathrm{C}, 320 \mathrm{~W}$ and 5 minutes, respectively. After the experimental test at the optimum point, the number of E. coli was equal to zero, confirming the optimum point determined. It should be noted that energy consumption in the process was equal to 64.428 $\mathrm{kJ} / \mathrm{L}$, which the share of energy consumption of ultrasound device is negligible compared to the microwave. The amount of energy consumption can be approximated the desired value with changes in waves on the microwave, giving rise to focusing the waves.

\section{Discussion}

When the temperature increased to $50^{\circ} \mathrm{C}$ by increasing the microwave power, the reduction of E. coli was higher

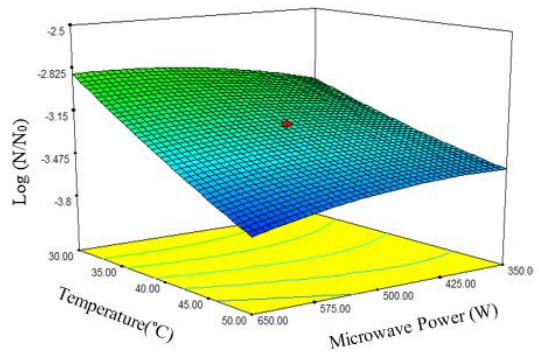

(b)

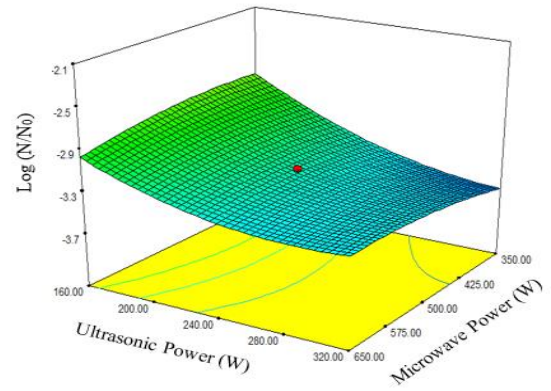

(d)

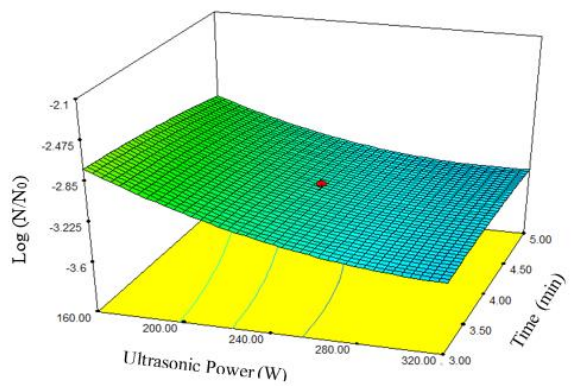

(f)

Figure 4. Changes in the number of log cycles of the reduction of $E$. coli and ERE influenced by $a, b)$ temperature and microwave power $\mathrm{c}, \mathrm{d})$ ultrasound power and microwave power $\mathrm{e}, \mathrm{f}$ ) ultrasound power and treatment time $\mathrm{g}, \mathrm{h}$ ) ultrasound power and temperature. 
Table 4. Boundary conditions for optimizing of the process

\begin{tabular}{|c|c|c|c|c|c|c|}
\hline Name & Goal & Lower limit & Upper limit & Lower weight & Upper weigh & Importance \\
\hline Microwave power (W) & In range & 350 & 650 & 1 & 1 & 3 \\
\hline Temperature $\left({ }^{\circ} \mathrm{C}\right)$ & In range & 30 & 50 & 1 & 1 & 3 \\
\hline Ultrasonic power (W) & In range & 160 & 320 & & 1 & 3 \\
\hline Ultrasonic exposure time (min) & In range & 3 & 5 & 1 & 1 & 3 \\
\hline E-coli count & Target & -5 & $2.1008-$ & 1 & 1 & 5 \\
\hline Energy (KJ) & Minimize & 13.044 & 53.0525 & 1 & 1 & 3 \\
\hline ERE & Maximize & 0.0645226 & 0.200751 & 1 & 1 & 4 \\
\hline
\end{tabular}

than the other treatments. This was most probably due to the higher speed of temperature increase in which the microorganisms have not enough time to adapt to the new conditions (16).

By increasing the ultrasound power, the lethal effects of ultrasound increased. The reason for the E. coli reduction can be the increased motion range of the ultrasonic horn in the fluid. The increased motion range causes to increase the number of bubbles formed in the fluid and thereby an increase in the cavitation action (17).

Increasing the treatment time causes to increase the number of audio streams periods in the reactor and consequently, the profound effect of the ultrasound waves on E. coli increased. Similar results were reported by researchers in the case of other fruit juices $(12,18)$.

It should be noted that with respect to the slope of the curve along the axes of temperature and time, the effect of temperature in E. coli reduction was higher than the effect of the treatment time with ultrasound. Similar results have been reported in other studies $(12,18)$.

The mechanism of microorganisms' inactivation is primarily based on the physical and chemical factors caused by the effect of ultrasound on liquid foods. Among the physical effects, thinning the cell membranes and producing local heat and pressure changes $\left(5500^{\circ} \mathrm{C}\right.$ and $50000 \mathrm{kPa}$ ), and among the chemical effects, producing the free radicals such as hydroxyl and hydrogen radicals caused by sonochemical reactions are considered $(19,20)$. Therefore, it can be deduced that achieving the acceptable levels of inactivation was due to the simultaneous effect of ultrasound waves and heat generated by ultrasound (21). Furthermore, the ultrasound treatment conditions such as frequency, intensity, the location of the horn in the reactor, the geometric characteristics of the horn and the reactor, the method and location of sampling, the density of sound energy, and the environmental characteristics have important roles in determining the speed of inactivation (22). In addition, the effectiveness and efficiency of ultrasound in the inactivation of microorganisms is under the influence of physicochemical properties, the volume of food, processing temperature, intensity and duration of ultra-sonication (19).

In this study, the effect of microwave and ultrasound on the chemical and qualitative properties of grape juice was not studied. However, the literature review shows that the mechanism of microorganisms' inactivation, which is essentially based on the physical and chemical characteristics of products, has different effects on the chemical and qualitative properties of products. Their chemical effects are free radicals such as hydroxyl and hydrogen radicals $(20,23)$.

In a study, it was shown that any increase in the microwave power, the ultrasonic power, and ultra-sonication time resulted in a significant decrease in the vitamin $\mathrm{C}$ content in cherry juice but the effect of the ultrasonic power was greater than the other factors (10).

In a research, the combined effect of ultrasound, heat and light pulses on the inactivation of $E$. coli and qualitative characteristics of apple juice was studied. In this study, the effects of four levels of energy, and light pulses, heat and ultrasound were investigated, which the highest amounts of destroying microorganisms were for ultrasonic, temperature and light pulses, respectively. All treatments caused to change the color of apple juice. The order of the applied treatments had a significant effect on changing the color of the juice. It was also found that different levels of applied energy were ineffective in measuring the qualitative attributes of the apple juice (24).

In a study on orange juice, a significant reduction in the amount of ascorbic acid as a function of sound energy density and ultra-sonication time was done. It was reported that the amount of decreased ascorbic acid was less than $5 \%$ in the higher density and time applied. In addition, in this study, it was found that the amount of residual ascorbic acid using ultrasound was better than the conventional thermal methods (25).

\section{Conclusion}

Microwave power, fruit juice temperature, ultrasound power, and ultrasonic exposure time were effective in the number of E. coli and ERE. The importance of the final temperature of the grape juice in reduced $E$. coli was higher than the microwave power that causes the temperature. The effect of microwave power on ERE was more than the effect of temperature. The ultrasound power was more effective in reducing E. coli in grape juice as compared to 
the microwave power. In addition, the reduction of the microbial number in a cycle for each $\mathrm{kJ}$ of ultrasound energy increased as compared microwave energy. With increasing the duration of treatment with ultrasound, the number of $E$. coli in grape juice decreased. The increased duration of treatment increased the number of periods of audio streams in the reactor and the effect of ultrasound waves on E. coli. In addition, increased consumption of the ultrasound energy was more effective in reduction of the microbial number in a cycle as compared to increased ultrasound treatment time and increased ultrasound power consumption. Both sample temperature and ultrasonic duration were important independent variables and effective factors on E. coli reduction. Finally, the combination method can decrease E. coli count successfully, so the authors suggest the mentioned method in food processing.

\section{Acknowledgements}

Research Council of Shahrekord University of Medical Sciences is thankfully acknowledged for the financial support to carrying out the work (grant No. 2217). Authors are also thankful to Research Council of Shahrekord University for the provided supports.

\section{Authors' contributions}

BHS and ZL contributed to design of the study, supervised the research and manuscript editing, SR and HZ helped the supervision and preparation of the manuscript. $\mathrm{MB}$ and $\mathrm{AB}$ performed the experiments, data collection and prepare manuscript drafting. All authors read and confirmed the final version of the manuscript for publication.

\section{Conflict of interests}

The authors declared no competing interests.

\section{Ethical considerations}

Ethical issues in research have been completely observed by the authors.

\section{Funding/Support}

This research was financially supported by Shahrekord University of Medical Sciences, Shahrekord, Iran (Grant No. 2217).

\section{References}

1. Kuldiloke J. Effect of ultrasound, temperature and pressure treatments on enzyme activity and quality indicators of fruit and vegetable juices [Thesis]. Berlin: Institute of Food Technology Food Biotechnology and Process Technology, the Technical University of Berlin; 2002.

2. Hosseinzadeh Samani B, Khoshtaghaza MH, Minaei S, Zareifourosh H, Eshtiaghi MN, Rostami S. Design, development and evaluation of an automatic fruit-juice pasteurization system using microwave - ultrasonic waves. J Food Sci Technol. 2016;53(1):88-103. doi: 10.1007/s13197-015-2026-6.

3. Vikram VB, Ramesh MN, Prapulla SG. Thermal degradation kinetics of nutrients in orange juice heated by electromagnetic and conventional methods. J Food Eng. 2005;69(1):31-40. doi: 10.1016/j.jfoodeng.2004.07.013.

4. Lee HS, Coates GA. Effect of thermal pasteurization on Valencia orange juice color and pigments. LWT Food Sci Technol. 2003;36(1):153-6. doi: 10.1016/S00236438(02)00087-7.

5. Neis U, Blume T. Ultrasonic disinfection of wastewater effluents for high-quality reuse. Water Science and Technology: Water Supply. 2003;3(4):261-7.

6. Toepfl S, Heinz V, Knorr D. High intensity pulsed electric fields applied for food preservation. Chem Eng Process. 2007;46(6):537-46.

7. Katsaros GI, Katapodis P, Taoukis PS. Modeling the effect of temperature and high hydrostatic pressure on the proteolytic activity of kiwi fruit juice. J Food Eng. 2009;94(1):40-5. doi: 10.1016/j.jfoodeng.2009.02.026.

8. Koutchma T, Le-Bail A, Ramaswamy HS. Comparative experimental evaluation of microbial destruction in continuous-flow microwave and conventional heating systems. Can Biosyst Eng. 2001;43:3.1-3.8.

9. Yildiz H, Bozkurt H, Icier F. Ohmic and Conventional Heating of Pomegranate Juice: Effects on Rheology, Color, and Total Phenolics. Food Sci Technol Int. 2009;15(5):503-12. doi: 10.1177/1082013209350352.

10. Samani BH, Khoshtaghaza MH, Lorigooini Z, Minaei S, Zareiforoush H. Analysis of the combinative effect of ultrasound and microwave power on Saccharomyces cerevisiae in orange juice processing. Innov Food Sci Emerg Technol. 2015;32(Suppl C):110-5. doi: 10.1016/j. ifset.2015.09.015.

11. Valero M, Recrosio N, Saura D, Munoz N, Marti N, Lizama V. Effects of ultrasonic treatments in orange juice processing. J Food Eng. 2007;80(2):509-16. doi: 10.1016/j. jfoodeng.2006.06.009.

12. Wu J, Gamage TV, Vilkhu KS, Simons LK, Mawson R. Effect of thermosonication on quality improvement of tomato juice. Innov Food Sci Emerg Technol. 2008;9(2):186-95. doi: 10.1016/j.ifset.2007.07.007.

13. Lorigooini Z, Samani BH, Zareiforoush H. Optimization of the Efficiency of Electromagnetic Waves Dryer Power on Chemical Composition and Yield of Satureja bachtiarica Essential Oil Using Response Surface Methodology. Journal Essential Oil Bearing Plants. 2017;20(1):1-11. doi: 10.1080/0972060X.2016.1264277.

14. Hosseinzadeh Samani B, Khoshtaghaza MH, Minaee S, Abbasi S. Modeling the Simultaneous Effects of Microwave and Ultrasound Treatments on Sour Cherry Juice Using Response Surface Methodology. J Agric Sci Technol. 2015;17(4):837-46.

15. Hemmatian R, Najafi G, Hosseinzadeh B, Tavakkoli Hashin T, Khoshtaghaza MH. Experimental and Theoretical Investigation of the Effects of Moisture Content and Internodes Position on Shearing Characteristics of Sugar Cane Stems. J Agric Sci Technol. 2012;14(5):963-74. 
16. Tajchakavit S, Ramaswamy HS, Fustier P. Enhanced destruction of spoilage microorganisms in apple juice during continuous flow microwave heating. Food Res Int. 1998;31(10):713-22. doi: 10.1016/S0963-9969(99)000502.

17. Vichare NP, Gogate PR, Dindore VY, Pandit AB. Mixing time analysis of a sonochemical reactor. Ultrason Sonochem. 2001;8(1):23-33.

18. Ugarte-Romero E, Feng H, Martin SE, Cadwallader KR, Robinson SJ. Inactivation of Escherichia coli with Power Ultrasound in Apple Cider. J Food Sci. 2006;71(2):E102-E8. doi: 10.1111/j.1365-2621.2006. tb08890.x.

19. Piyasena P, Mohareb E, McKellar RC. Inactivation of microbes using ultrasound: a review. Int J Food Microbiol. 2003;87(3):207-16.

20. Valdramidis VP, Cullen PJ, Tiwari BK, O'Donnell CP. Quantitative modelling approaches for ascorbic acid degradation and non-enzymatic browning of orange juice during ultrasound processing. J Food Eng. 2010;96(3):449-54. doi: 10.1016/j.jfoodeng.2009.08.025.

21. Lee H, Zhou B, Liang W, Feng H, Martin SE. Inactivation of Escherichia coli cells with sonication, manosonication, thermosonication, and manothermosonication: Microbial responses and kinetics modeling. J Food Eng. 2009;93(3):354-64. doi: 10.1016/j.jfoodeng.2009.01.037.

22. Lee $H$, Zhou B, Feng $H$, Martin SE. Effect of $\mathrm{pH}$ on inactivation of Escherichia coli K12 by sonication, manosonication, thermosonication, and manothermosonication. J Food Sci. 2009;74(4):E191-8. doi: $\quad$ 10.1111/j.1750-3841.2009.01130.x.

23. Gomez-Lopez VM, Orsolani L, Martinez-Yepez A, Tapia MS. Microbiological and sensory quality of sonicated calcium-added orange juice. LWT Food Sci Technol. 2010;43(5):808-13. doi: 10.1016/j.lwt.2010.01.008.

24. Munoz A, Caminiti IM, Palgan I, Pataro G, Noci F, Morgan DJ, et al. Effects on Escherichia coli inactivation and quality attributes in apple juice treated by combinations of pulsed light and thermosonication. Food Res Int. 2012;45(1):299-305. doi: 10.1016/j.foodres.2011.08.020.

25. Tiwari BK, O’ Donnell CP, Muthukumarappan K, Cullen PJ. Effect of sonication on orange juice quality parameters during storage. Int J Food Sci Technol. 2009;44(3):586-95. doi: 10.1111/j.1365-2621.2008.01858.x. 